\title{
HispanismeS
}

Revue de la Société des Hispanistes Français

\section{Escrituras. Collaborations artistiques dans l'espace urbain en tension}

Escrituras. Colaboraciones artísticas en el espacio urbano en tensión

Artistic collaborations in the urban space in tension

\section{Diego Jarak}

\section{(2) OpenEdition}

Journals

Édition électronique

URL : https://journals.openedition.org/hispanismes/406

DOI : 10.4000/hispanismes.406

ISSN : 2270-0765

Éditeur

Société des Hispanistes Français

Référence électronique

Diego Jarak, « Escrituras. Collaborations artistiques dans l'espace urbain en tension », HispanismeS

[En ligne], 14 | 2019, mis en ligne le 01 octobre 2019, consulté le 31 août 2021. URL : http://

journals.openedition.org/hispanismes/406 ; DOI : https://doi.org/10.4000/hispanismes.406

Ce document a été généré automatiquement le 31 août 2021.

Les contenus de cette revue sont mis à disposition selon les termes de la Licence Creative Commons Attribution - Pas d'Utilisation Commerciale - Pas de Modification 4.0 International. 


\title{
Escrituras. Collaborations artistiques dans l'espace urbain en tension
}

\author{
Escrituras. Colaboraciones artísticas en el espacio urbano en tensión \\ Artistic collaborations in the urban space in tension
}

Diego Jarak

\section{Introduction}

1 Escrituras est un projet collectif, non seulement parce qu'il a été imaginé et mis en œuvre par deux artistes, Mariela Yeregui et Gabriela Golder, avec le soutien d'autres artistes invités, mais aussi parce qu'il est construit sur le territoire, et même plus, avec le territoire.

2 Escrituras est une série d'actions, d'interventions et de déambulations dans l'espace. Escrituras est aussi une série d'installations lumineuses composées de six affiches textuelles en Néon installées sur les façades de certains bâtiments et autres espaces publics.

3 Escrituras est né d'une recherche commune des deux artistes et de la volonté d'interroger l'espace public à partir de dispositifs et techniques de dérivations psychogéographiques $^{1}$ et de ressources de représentation graphique et textuelle. Selon les artistes, «Escrituras es un proyecto de producción comunitaria, participativa y performativa y de intervención territorial sobre la base de estrategias cartograficas open-source $»^{2}$.

4 Enfin, Escrituras est un projet qui a été développé dans le cadre d'un appel à projets lancé par le gouvernement de la ville de Buenos Aires et, en ce sens, un projet avec une dimension politique incontournable. En intervenant dans l'espace public avec un financement public et selon les clauses d'un marché public (stipulées dans les 
conditions et le règlement de l'appel à projets), le travail des artistes est nécessairement en dialogue avec ce cadre institutionnel d'État.

Cela dit, ce qui nous intéresse dans cette initiative originale à bien des égards, c'est d'analyser les stratégies mises en place par les artistes pour développer leur projet, alors même qu'elles sont allées à l'encontre de ce que précisément, l'appel ne disait pas mais laissait présumer.

6 Pour analyser cet artefact complexe qu'est le projet Escituras et son déploiement dans l'espace urbain nous allons, dans un premier temps, présenter le cadre dans lequel s'inscrit l'appel à projets et tout particulièrement le contexte de gentrification. Dans un second temps, nous présenterons les processus de l'œuvre, notamment ses aspects collaboratifs construits à partir d'une interprétation des déambulations psychogéographiques.

\section{Un appel à projet qui ne dit pas son nom}

7 En 2014, le gouvernement de la ville de Buenos Aires lance un appel à artistes, designers, architectes et créateurs afin d'intervenir dans cinq espaces de la ville. Le titre de l'appel était Buenos Aires - Sitio específico, en référence à la pratique artistique in situ ${ }^{3}$, qui consiste à créer des œuvres en dialogue direct avec un espace précis. Avec ce titre, le gouvernement de la ville joue avec trois niveaux de lecture. Dans un premier niveau, sûrement le plus évident, du moins pour les artistes, la résonance du titre avec la pratique du "site spécifique». Sur un deuxième plan, le titre servait de slogan publicitaire pour mettre en valeur les avantages de la ville en tant que destination touristique. «Buenos Aires - site spécifique » « Buenos Aires - ville unique ». Enfin, dans un troisième niveau de lecture, le titre et l'initiative sont venus confirmer un engagement politique qui a suscité des doutes, notamment parmi les associations de quartiers concernées.

8 Il s'agissait ici de réaffirmer un processus par lequel le gouvernement de Mauricio Macri cherchait, disait-on, à revitaliser certains secteurs défavorisés dans le sud de la ville ${ }^{4}$. Ainsi, la loi $n^{\circ} 4.353 / 12$ créa un territoire non imposable (paradis fiscal) pour les activités liées aux arts visuels, musicaux, littéraires et du spectacle vivant. Des activités donc que le gouvernement se permettait de regrouper sous une dénomination bien à la mode, celle des industries culturelles. Par ailleurs, le terme sous lequel ils inscrivaient le projet était encore un autre terme à la mode, celui de cluster ${ }^{5}$. Enfin, une des caractéristiques de ces clusters culturels était l'exemption de l'impôt sur le revenu brut et de l'impôt municipal ABL pendant 10 ans, de l'impôt et du paiement du droit de délimitation et de construction. Un investissement de l'État donc pour aider les futures entreprises à s'installer dans un quartier dans lequel plus de la moitié de la population vivait en dessous du seuil de pauvreté6.

9 Revitaliser les quartiers qui étaient tombés dans l'oubli des politiques gouvernementales pendant des décennies, paradoxalement, pouvait apparaître comme une démarche à caractère social. Or dans les orientations du gouvernement Macri une politique d'une telle nature posait questions. Plusieurs syndicats et organisations de quartier ont manifesté leurs craintes, comme l'explique Carlos Rodríguez dans un article publié dans le journal Pagina/12 le 24 octobre 2016, où il est déjà question de déloger les habitants historiques du quartier. Rodríguez écrit, à juste titre, que «le danger des expulsions » est un problème historique dans le quartier de La Boca, une 
forme de pression permanente qui pèse sur les habitants. Cependant, Rodríguez explique que ce processus a été totalement accéléré depuis la création (par le gouvernement de la ville) du District des Arts. Cette accélération, toujours selon Rodríguez, est dû au fait que

Los beneficios del Distrito gravan sobre los inmuebles y no sobre las actividades culturales, como debería ser, de manera que hay todo un mercado de especulación inmobiliaria que se volcó a comprar durante todo el proceso que terminó con la creación del Distrito ${ }^{7}$.

10 C'est dans ce contexte trouble ${ }^{8}$ que l'appel à projets d'intervention artistique a été publié, car si au moment du lancement de l'appel le processus d'expulsion - présenté par le gouvernement comme une redynamisation des quelques quartiers historiques oubliés par les politiques précédentes - était déjà en marche, ce n'était que le début de ces nouveaux clusters. Aussi, les voix d'opposition contre ce projet n'étaient-elles pas encore organisées non plus.

11 Ce qui nous intéresse c'est de comprendre comment le projet Escrituras s'est positionné artistiquement par rapport aux enjeux politiques et comment consciemment ou inconsciemment les artistes ont apporté leur grain de sable au débat. Il faut rappeler que le projet allait être financé et donc suivi par le gouvernement Macri, celui-là même qui mettait les habitants à la rue.

\section{Nommer pour mieux contrôler}

Un premier élément d'analyse concerne l'appel et la manière dont le gouvernement de la ville de Buenos Aires jouait déjà sur les mots pour faire avancer le projet. Ainsi, dans l'article 3 il était question d'identifier les espaces géographiques pour les futures interventions. Chaque espace était accompagné d'une nouvelle dénomination qui venait remplacer celles existant-avant le lancement de l'appel mais qui n'étaient utilisées que par les quelques secteurs de la classe moyenne liés au monde des arts et de la culture. Autrement dit, pour les habitants de ces quartiers ces dénominations étaient incompréhensibles 9 . Non seulement elles ne leur appartenaient pas mais ils ne percevaient pas non plus le lien direct ou indirect entre la dénomination et les espaces qu'ils habitaient. Ce premier niveau de contrôle, à première vue inoffensif, jouait pourtant un rôle important dans la construction d'une représentation de ce que le quartier allait devenir ${ }^{10}$, selon les bonnes intentions du gouvernement. Le langage utilisé pour nommer ces espaces, "clusters dynamiques et contemporains ", était issu d'une terminologie inspirée des modèles des villes et des quartiers nord-américains, plus connus pour leur taux de violence, de ségrégation et de stigmatisation que comme modèles d'intégration ou de transformation réussis.

13 En effet, les futurs espaces d'intervention artistique étaient précédés de la dénomination « District ». Pour les habitants des quartiers historiques de Buenos Aires, cette dénomination ne correspondait à aucune représentation physique, politique, économique ou culturelle. Une forme donc de déposséder les habitants de leurs espaces avant même que les lois du marché immobilier fassent leurs opérations. Ainsi, par ce geste simple mais très efficace, les quartiers n'appartenaient plus aux habitants, car ils n'existaient pas non plus ou ils cessaient d'exister comme tels ; désormais on parlerait de « District». 
Les cinq espaces identifiés dans l'appel étaient : 1- boulevard Benito Pérez Galdós (La Boca), District des Arts ; 2- boulevard Iriarte (Caseros), District du Design ; 3- Parc Patricios (Parque Patricios), District Technologique; 4- Square Jorge Newery (Chacarita), Quartier de l'Audiovisuel; 5- Florida et Sarmiento (4 esquinas), Microcentro. Force est de constater que tous les secteurs ou quartiers, à l'exception du " microcentro ", portaient un nom de métier. Par cet appel, la municipalité a cherché à renforcer l'orientation ou la caractérisation de certains quartiers, en suivant les orientations des tendances qui commençaient plus ou moins à se dessiner du fait de l'implantation de quelques artistes, développeurs, designers, etc. Autrement dit, dans le regard et la représentation de certains secteurs, une transformation de revalorisation était déjà en marche. La caractérisation des quartiers par le gouvernement a été voulue «neutre» en ce sens qu'elle s'inspire des environnements existants, à partir d'initiatives indépendantes et, disaient-ils ${ }^{11}$, des dynamiques associatives. Il s'agissait de donner une réponse officielle, institutionnelle et politique à un phénomène apparu de manière plus ou moins spontanée, mais surtout d'affirmer, puis de tenter d'établir un rythme, voire d'accélérer un processus qui était déjà en cours.

15 Le Ministre de l'époque chargé du Développement économique de la ville de Buenos Aires, Francisco Cabrera, déclarait « las grandes ciudades del mundo desarrollan sus industrias estratégicas y mejoran los barrios abandonados gracias a políticas como [la de la ley $4.353 / 12] »^{12}$. Un processus donc qui avait émergé avec sa propre logique, dans un contexte de globalisation et d'homogénéisation des villes, avec son propre dynamisme. L'État ne faisait qu'accompagner ces mouvements internationaux, comme quelque chose de supranational, qui le dépassait et de ce fait se justifiait de lui-même. Un phénomène de gentrification avec une logique propre qui comme le disait le Ministre « existe dans d'autres capitales du monde $»^{13}$.

En voulant inscrire les principes de la loi 4.353/12 dans ce cadre global, d'un mouvement bien plus large et commun à toutes les grandes villes, ce que Saskia Sassen appelle, à juste titre, "global city", Cabrera effaçait instantanément toutes les spécificités locales, et en particulier l'histoire du quartier. Mais les déclarations du Ministre ne sont pas un cas isolé dans ce paysage de transformations violentes, plutôt la règle qui s'impose comme le discours hégémonique depuis plusieurs décennies. Toujours d'après Sassen,

we can characterize the relationship of advanced to traditional capitalism in our current period as one marked by extraction and destruction, not unlike the relationship of traditional capitalism to precapitalist economies. At its most extreme this can mean the immiseration and the exclusion of growing numbers of people who cease being of value as workers and consumers ${ }^{14}$.

Or, en observant l'emplacement des lieux de l'appel sur une carte, la première chose qui attire l'attention est la concentration géographique, en particulier la proximité des trois premiers lieux. Le deuxième élément, toujours dans le registre spatial, est précisément le secteur sur lequel l'intérêt du gouvernement s'est concentré. Ces deux aspects s'expliquent par la même logique de réactivation de ces quartiers populaires, tombés dans l'oubli des politiques publiques. Transformer ces secteurs par l'introduction d'une aide et favoriser les industries culturelles, tel était l'objet annoncé par le gouvernement. L'appel à projets Buenos Aires - Sitio específico venait, d'une certaine manière, réaffirmer cette volonté et, dans la mesure du possible, lui donner un sens. Cependant, comme nous le verrons dans cet article, les effets secondaires ne 
seront pas sans conséquence, surtout pour les premiers intéressés, les habitants de ces quartiers.

L'élection de Mauricio Macri à la tête du gouvernement de la ville de Buenos Aires accélère le processus de gentrification de ces quartiers, comme le montre l'exemple de l'ancienne usine Alpargatas transformée peu de temps après en ensemble de logements de luxe (type loft), dans lequel le gouvernement lui-même avait installé quelques bureaux. Le rapprochement entre le marché public, l'initiative privée et le fait que le gouvernement installe ses propres bureaux passa inaperçu. D'une certaine manière, l'appel à projets «Buenos Aires - sitio específico » est apparu comme un écran de fumée pour cacher ces processus mais également pour les légitimer. La preuve en est que sur les cinq projets retenus, seuls deux ont été réalisés, un a été abandonné et les deux autres ont disparu des radars, avec le financement.

\section{Un projet entre deux rives}

19 Le projet Escrituras avait été sélectionné pour intervenir dans le District des Arts. Un secteur du Barrio de la Boca qui se caractérise par ses forts contrastes, situé dans le triangle entre les rues Blanes, Villafañe et l'autoroute. Au nord, le district est limitrophe de Catalinas, un complexe de logements de classe moyenne, avec un poumon vert, des parcs et une qualité de construction qui contraste avec les usines et les maisons du District des Arts. A l'ouest, le District borde la partie la plus touristique de La Boca, le passage caminito, avec ses bars, boutiques et restaurants.

20 La Boca, et cela est valable pour les deux autres quartiers Sud de Buenos Aires, est un espace qui, tout au long de son histoire, a été en tension permanente. Aujourd'hui, c'est un quartier où les conflits persistent en raison de la diversité des groupes sociaux qui y vivent. En effet, les zones défavorisées coexistent avec d'autres zones de classe moyenne et, plus récemment, avec des groupes sociaux de secteurs aisés. Très éclectique et hétérodoxe, c'est une zone en cours de transformation et, comme dans tout lieu gentrifié, ce processus implique une violence implicite et explicite.

21 Yeregui m'explique que, dans le secteur prédéterminé où [elles] allaient intervenir cinq pâtés de maisons autour de l'avenue Pérez Galdós -, il y avait aussi une forme de rupture sociale, semblable à un fossé. Pour elle, l'avenue Galdós est une artère centrale qui divise ce que certains habitants veulent séparer. Ainsi, lors des interventions elle aurait entendu un de ces voisins déclarer : « de este lado somos una cosa, del otro lado son otra cosa $»^{15}$. Cette avenue, poursuit Yeregui, est une fissure géante, comme un canal qui dissocie ce que chacun de ses habitants pense de son propre territoire et de l'Autre. A l'époque, conclut Yeregui, « [con Gabriela Golder] nos dijimos que el desafío era enorme: se trataba de generar una dinámica de grupo, de comunidad, en un espacio en fricción ${ }^{16}$. Les artistes proposent ainsi et dès le départ une relecture de l'ambiance du quartier à partir d'une série de stratégies qui se rapprochent de la lecture que faisaient les situationnistes.

22 Le projet Escrituras cherchait ainsi à activer des espaces de mémoire individuelle et collective de ce lieu commun et partagé, le quartier de la Boca, et en particulier le Distrito de las Artes. Il proposait un aller-retour sur le territoire à la recherche de ceux qui révèlent les symptômes dont le quotidien est témoin et complice. Dans les termes de Guy Debord, il s'agissait de «trouver les issues psychogéographiques d'une zone en 
s'écartant systématiquement de tous les points coutumiers. On peut alors s'égarer dans des quartiers déjà fort parcourus $»^{17}$.

Le résultat, six panneaux en néon, sont le fruit d'un travail collaboratif qui s'est développé pendant plusieurs mois dans une série d'ateliers, rencontres, débats, déambulations avec les habitants du quartier. Ainsi, si les œuvres des deux artistes se caractérisent par un recours à la technologie ${ }^{18}$ que Yeregui s'amuse à définir comme un « lieu de rencontre », l'utilisation et le recours au néon, « una tecnología del pasado que está en vías de desaparición $»^{19}$, apparaît alors comme une référence et une critique directe du mode de production capitaliste - une critique des modes d'appropriation de la technologie et non des technologies elles-mêmes. Et, nous le verrons plus loin, leur démarche artistique se veut aussi une manière de rompre avec ce qu'Yves Bonard appelle « l'impossible réappropriation de l'espace urbain par l'imaginaire ${ }^{20}$.

\section{La Boca, espace urbain en tension}

"Más allá de los recuerdos se trataba de articular acciones concretas para que lo invisible se vuelva visible $\aleph^{21}$. Pour les artistes, il s'agissait d'activer une forme de mémoire qui renvoie au futur et qui souligne que, là où il y a de la place pour la mémoire, il y a aussi de la place pour la conscience active. C'est dans ce registre que le projet réagit aux attentes cachées de l'appel à projet et c'est aussi à ce niveau que la démarche de déambulation peut être lue comme une invitation à la révolte.

En effet, le projet Escrituras conçoit un type d'œuvre qui expérimente la mémoire historique du quartier d'une manière non figée, en partant du principe que l'articulation des actes de mémoire est un processus progressif et dynamique. «Ce n'est pas que le passé jette sa lumière sur le présent, ou que le présent jette sa lumière sur le passé $~_{22}$, écrit Walter Benjamin dans les textes préparatoires des thèses sur l'histoire. Pour l'auteur des passages de Paris, «l'image est plutôt ce en quoi le passé converge avec le présent en une constellation ${ }^{23}$. C'est dans ce mouvement entre passé et présent que le Projet Escrituras cherche à activer des formes de révolte, par et dans la création. "Tandis que la relation entre l'alors et le maintenant est purement temporelle - continue Benjamin -, la relation du passé avec le présent est dialectique, par bonds $»^{24}$. Cela veut dire que, pour Benjamin, passé et présent se reconnaissent mutuellement dès lors que le présent se reconnait signifié dans le passé et que le passé trouve son accomplissement dans le présent.

Pour Yeregui, "la idea era de recorrer les distintos corredores sociales, políticos, culturales e históricos desde diferentes puntos de vista y perspectivas ${ }^{25}$. Il ne s'agissait donc pas d'actes de mémoire circonscrits à la sphère de l'évocation, mais d'une volonté d'abolir toute tentative de pétrification de la mémoire par l'activation d'un acte de pensée, "qui entraînerait un acte de faire partie, d'un "être-devenir" sur le territoire concret $»^{26}$.

Il n'était donc pas question d'intervenir dans l'espace public du point de vue des artistes, mais de composer avec l'espace et ce qui s'y passe, dans un dialogue direct, un corps à corps, avec ce que la mémoire pouvait faire émerger dans le cadre des activations proposées. Yeregui et Golder ont opté pour une intervention/occupation du territoire afin de mettre en lumière les tensions et les frictions. Elles ont proposé une série d'ateliers participatifs et communautaires animés par des amis artistes et des invités. Force est de constater l'importance que les artistes donnent à une certaine idée 
de communauté et qui s'inscrit directement dans la démarche artistique du projet. Une communauté d'artistes-amis pour travailler à partir de différentes ressources et de différentes sources afin de créer une plateforme collaborative et co-construite. Une sorte d'archive vivante de la mémoire actuelle du Barrio, un développeur de frictions, un artefact de visibilisation et d'invisibilité à l'usage des voisins. Une plateforme qui a pris la forme d'un site internet ouvert et collaboratif où tout le monde pouvait déposer ses apports.

\section{Mettre en lumière les frictions}

La visibilisation de l'espace du quartier était basée sur quatre axes de nature diverse : sonorité, écriture, corporalité et visualisation. Yeregui explique que "los paisajes sonoros activan la percepción de la escucha para fijar los sonidos que generalmente son inaudibles $»^{27}$. D'autre part, poursuit Yeregui, « el silencio interior y la atención calma de los participantes abrieron posibilidades y reflexiones sobre la identidad y la territorialidad $\aleph^{28}$. Dans ce travail du détail, qui s'insère dans les interstices et les fissures ouvertes par un processus d'institutionnalisation et d'accélération de l'embourgeoisement du quartier, les artistes-communauté-amis ont créé une nouvelle temporalité. Celle de l'onomatopée et des murmures, «los sonidos y los ruidos naturalizados fueron puestos de relieve a través de las deambulaciones psicogeográficas que permitieron la construcción de metáforas sonoras $»^{29}$, à partir d'une réflexion sur l'évidence comme résultat d'un regard et d'un langage automatiques, comme vide dans la représentation, que l'on suppose être connu et qu'il est donc inutile de nommer.

Les déambulations psychogéographiques ont été largement inspirées par les situationnistes. Ces actions ne sont pourtant pas sans rapport avec le romantisme. La position des premiers romantiques, en effet, peut être considérée comme oscillant entre le dépouillement et l'enthousiasme, d'où la nécessité d'une expérience du monde que la pensée rationnelle semblait avoir confisquée. Romantisme et rationalisme apparaissent alors comme les deux faces d'une même pièce. La conquête du monde par l'application des outils mathématiques à la technique puis, aujourd'hui, à la technologie afin de mettre en lumière les processus de la nature, répondait, du côté du romantisme, à la nécessité d'une restitution du monde par le biais du rêve considéré à cette époque comme un nouvel outil permettant l'accès à l'inconscient.

La dissolution de l'ego, au fondement de cet autre processus de connaissance, apparait comme la condition d'émergence d'un inconscient conçu comme devenir de la nature du monde et de l'histoire. Les situationnistes, de leur côté, critiquaient la privatisation de l'expérience ce qui les avaient conduits à proposer de nouvelles formes de pratiques dont l'enjeu était la réappropriation du désir en tant qu'expression de la singularité de chacun. Cette réappropriation, justement, prit la tournure d'une série de propositions exploratoires de l'environnement répondant à un projet de cartographie, réintroduisant la notion de territoire. Ainsi Debord écrivait en 1956,

[...] il existe un relief psychogéographique des villes, avec des courants constants, des points fixes, et des tourbillons qui rendent l'accès ou la sortie de certaines zones fort malaisées [...]. L'analyse écologique du caractère absolu ou relatif des coupures $\mathrm{du}$ tissu urbain, du rôle des microclimats, des unités élémentaires entièrement distinctes des quartiers administratifs, et surtout de l'action dominante des centres d'attraction, doit être utilisée et complétée par la méthode psychogéographique ${ }^{30}$. 
31 classer et révéler des phrases cachées dans le paysage, à partir de la constitution d'une base de données dont l'objet est la rue elle-même. L'inscription du corps dans l'environnement, pour sa part, a été explorée dans une série de pratiques corporelles à travers lesquelles les participants ont reconnu de nouvelles voies et de nouvelles conceptions spatiales. Des stratégies tendant à invisibiliser le corps dans les architectures ont été créées pour explorer les façons de le traverser en groupe. Dans la tension de l'invisible avec le visible, il y a un effet de distanciation par lequel le corps est réintroduit dans sa propre territorialité. En ce qui concerne la visualisation, les artistes ont proposé une série de pratiques pour marquer les lieux cachés. Puits, fissures, trous dans un mur, sont des traces qui suggèrent de nouvelles voies et des conceptions spatiales. En rompant les conventions de parcours prédéterminés, elles ont défini d'autres connexions entre les espaces pour créer un nouveau territoire visuel et textuel.

Dans chacun des ateliers, il y avait un espace de réflexion dans lequel des textualités se posaient par rapport à l'espace. La nouvelle cartographie s'est cristallisée dans une discursivité produite collectivement. Ces matériaux textuels, générés au cours des expériences de déambulation, ont fait l'objet de débats qui, à leur tour, ont déclenché de nouvelles discursivités à la suite de la cartographie collective lors des rencontres post-ateliers. Tous ces documents visuels et textuels ont été inclus dans leur support original sur le site Web du projet.

Les phrases qui envisageaient d'autres façons de concevoir le territoire et les relations qui s'y imbriquaient ont été choisies en groupes. Il s'agit de : «Volvernos invisibles»; «Se corrió el sol y cambió el límite »; «Es imposible el silencio »; « El viento arrasa y se va »; «El terreno se vuelve a mover » et «Eternos por hora, por día, por mes ». Elles forment un récit visuel, spatial, urbain, lumineux et aérien sous forme d'affiches, composant un récit poétique à caractère collectif. Ces phrases ont été transposées dans de grandes structures de néon qui ont été placées sur les toits des bâtiments situés sur la rue où les déambulations ont eu lieu. Ainsi, la signalétique urbaine, intégrée à l'architecture de l'usine et de l'habitat, construit une écriture spatiale à partir des pratiques narratives de la communauté déployées lors des ateliers. Il s'agissait de transcender la cartographie canonique, c'est-à-dire les formes standardisées de représentation territoriale, et de faire du territoire le véritable cadre des dialogues possibles, ouvrant un horizon où l'action et la construction collectives susciteraient de nouveaux débats autour du territoire. Il existe des points fixes dont la mémoire est préservée à travers différentes formes culturelles - images, récits épiques, rites, textes, etc. - ; ce sont des îlots de temps qui rendent possible l'objectivation de la culture. Il y a des faits concrets et réels, qui sont déconstruits en différentes visualités et sonorités dans nos mémoires. Ces vestiges sont liés à des discursivités - historiques, sociales et culturelles - qui insèrent l'évènement réel dans une chaîne plus large. C'est le fragment, en somme, qui façonne la mémoire collective. Comme le dit KourtessiPhilippakis «le territoire est à la fois objectivement organisé et culturellement inventé $\aleph^{31}$. 


\section{NOTES}

1. La psychogéographie "proposerait l'étude des lois exactes, et des effets précis du milieu géographique, consciemment aménagé ou non, agissant directement sur les émotions et le comportement des individus", Guy DEBORD, "Introduction à une critique de la géographie urbaine ", Les lèvres nues, Bruxelles, n 6, 1955, <URL : https://www.larevuedesressources.org/ introduction-a-une-critique-de-la-geographie-urbaine,033.html> [Consulté le 10/09/2020].

2. Entretien de Diego Jarak avec Mariela Yeregui à Buenos Aires le 22 juin 2019.

3. L'expression site-specific (in situ) désigne une œuvre qui a été conçue pour le site d'accueil. Ainsi, l'artiste qui pratique la création in-situ dédie son œuvre à un espace (que ce soit un espace public, comme c'est souvent le cas, mais aussi un espace privé - musée, galerie, etc. - car l'œuvre ne peut être transportée). L'œuvre «fonctionne » avec l'espace, l'espace d'accueil fait partie de l'œuvre.

4. Soledad ARQUeros et Carolina gonZALEZ REDONDo, «La política de distritos del sur de Buenos Aires : una mirada en perspectiva ", Quid 16 - Revista del area de estudios urbanos, Buenos Aires, $\mathrm{n}^{\circ}$ 7, jun.-nov. 2017, p. 7-29.

5. Selon Porter, les clusters sont des «Groupes géographiquement proches d'entreprises interconnectées et d'institutions associées dans un domaine particulier, unis par des éléments communs et complémentaires", Michel PORTER, "The competitive advantage of nations", Harvard Business Review, 1990, p. 73.

6. «Incidencia de la pobreza y la indigencia en 31 aglomerados urbanos », Informes técnicos, vol. 3 , $\mathrm{n}^{\circ}$ 182. $\quad$ <URL : https://www.indec.gob.ar/uploads/informesdeprensa/ eph_pobreza_01_19422F5FC20A.pdf> [Consulté le 12/10/2019].

7. Carlos RODRIGUEZ, «La Boca resiste y canta contra el desalojo », in Página 12 du 24/10/2016. <URL : https://www.pagina12.com.ar/diario/sociedad/3-312504-2016-10-24.html> [Consulté le 12/10/2019].

8. «La creación del distrito de las artes fue ampliamente resistida por parte de organizaciones barriales, vecinos y artistas de La Boca, que entendían que el proyecto favorecería la especulación inmobiliaria, incrementaría los valores del suelo y de los alquileres y terminaría expulsando a los sectores populares del barrio ; a la vez que no otorgaba ventajas económicas relevantes para los artistas históricos que residen y desarrollan sus actividades creativas en este sector de la ciudad » in Arqueros et González Redondo, op.cit., p. 27.

9. Ana Gretel thomazs, «Los nuevos distritos creativos de la Ciudad de Buenos Aires: la conversión del barrio de La Boca en el Distrito de las Artes », Revista Eure, 42, p. 145-167.

10. Le contrôle est désormais une relation de vitesse. Les changements s'opèrent rapidement, sans délai, de sorte qu'il n'y ait pas de temps de réaction possible. D'ailleurs, le temps des changements est devenu la norme. Tout change, tout le temps. Gilles Deleuze écrit: «Les enfermements sont des moules, des moulages distincts, mais les contrôles sont une modulation, comme un moulage auto-déformant qui changerait continûment, d'un instant à l'autre, ou comme un tamis dont les mailles changeraient d'un point à un autre.[...] Dans les sociétés de discipline, on n'arrêtait pas de recommencer (de l'école à la caserne, de la caserne à l'usine), tandis que dans les sociétés de contrôle on n'en finit jamais avec rien, l'entreprise, la formation, le service étant les états métastables et coexistants d'une même modulation, comme d'un déformateur universel », in Gilles DELEUZE, « Post Scriptum sur les sociétés de contrôle », L'autre journal, n 1, mai 1990.

11. Déclarations du Ministre au développement économique pour la ville de Buenos Aires, Francisco CABRERA, à Noticias urbanas, le 2 novembre 2012.

12. Ibid.

13. Ibid. 
14. Saskia SASSEN, Expulsions: Brutality and Complexity in the Global Economy, Cambridge, MA, USA, Harvard University Press, 2014, p. 10. («nous pouvons caractériser la relation du capitalisme avancé au capitalisme traditionnel dans notre période actuelle comme une relation marquée par l'extraction et la destruction, un peu comme la relation du capitalisme traditionnel aux économies précapitalistes. À l'extrême, cela peut signifier la paupérisation et l'exclusion d'un nombre croissant de personnes qui cessent d'être des travailleurs et des consommateurs de valeur » [traduction de l'auteur]).

15. Ibid.

16. Ibid.

17. Guy DEBORD, «Théorie de la dérive », Les lèvres nues, Bruxelles, $\mathrm{n}^{\circ}$ 9, décembre 1956.

18. Mariela Yeregui <URL: https://yereguimariela.wordpress.com/>; Gabriela Golder <URL: https://www.gabrielagolder.com/>.

19. Ibid.

20. Yves BONARD, «Dérive et dérivation. Le parcours urbain contemporain, poursuite des écrits situationnistes? ", Journal of Urban Research, n² 2, 2009, p. 9.

21. Entretien de Diego Jarak réalisé à Mariela Yeregui à Buenos Aires le 22 juin 2019.

22. Walter BENJAMIN, "Materiales preparatorios del escrito Sobre el concepto de histoira", Benjamin-Archiv, Ms 474 cité par Pablo scotTo BENITo «El materialismo histórico de Benjamin : tradición, detención y destrucción », Constelaciones - Revista de teoría crítica, n 7, Barcelona, diciembre 2015.

23. Ibid.

24. Ibid.

25. Entretien de Diego Jarak réalisé à Mariela Yeregui à Buenos Aires le 22 juin 2019.

26. Ibid.

27. Ibid.

28. Ibid.

29. Ibid.

30. DEBORD, « Théorie de la dérive », Op. cit.

31. Georgia KOURTESSI-PHILIPPAKIS, Introduction à Archéologie du territoire, de l'Égée au Sahara, Paris, Éditions de la Sorbonne, 2011, p. 9.

\section{RÉSUMÉS}

Escrituras est un projet collectif d'intervention dans l'espace urbain des artistes argentines Mariela Yeregui et Gabriela Golder. Le projet est une réponse à un appel lancé par le gouvernement de la ville de Buenos Aires, et dans le cadre d'un processus de gentrification impliquant les quartiers de l'appel. Le contexte politique et social du projet provient également du contexte géopolitique, puisque l'espace d'intervention est marqué par une longue histoire, notamment celle de l'immigration. L'article tente donc de positionner l'intervention urbaine comme un outil qui cherche à problématiser les tensions à travers une série d'ateliers participatifs et un processus de déambulation et de co-construction.

Escrituras is a collective project of intervention in urban space by Argentine artists Mariela Yeregui and Gabriela Golder. The project was born as a response to a call launched by the government of the city of Buenos Aires, and within the framework of a gentrification process 
involving the neighborhoods. The political and social context of the project also comes from the geopolitical background, since the space of the intervention is marked by a long history, particularly that of immigration. The article thus attempts to position urban intervention as a tool that seeks to question the tensions through a series of participatory workshops and coconstruction wandering processes.

Escrituras es un proyecto colectivo de intervención del espacio urbano de las artistas argentinas Mariela Yeregui y Gabriela Golder. El proyecto nace como respuesta a una convocatoria lanzada por el gobierno de la ciudad de Buenos Aires, y en el marco de un proceso de gentrificación del que participan los barrios de la convocatoria. El contexto político y social del proyecto viene además del trasfondo geopolítico, ya que el espacio de la intervención está marcado por una larga historia, y en particular aquella de la inmigración. El articulo intenta así posicionar la intervención urbana como una herramienta que busca problematizar las tensiones a partir de una serie de talleres participativos y de procesos deambulatorios de co-construcción.

\section{INDEX}

Mots-clés : art urbain, errance psychogéographique, site spécifique, Buenos Aires

Palabras claves : arte urbano, deambulación psicogeográfica, sitio específico, Buenos Aires

Keywords : urban art, psycho-geographical wandering, site-specific, Buenos Aires

\section{AUTEUR}

DIEGO JARAK

(CRHIA/La Rochelle Université) 